\title{
THE MEDIATING ROLE OF SELF-EFFICACY IN THE RELATIONSHIP BETWEEN WORKPLACE BULLYING, MENTAL HEALTH AND AN INTENTION TO LEAVE AMONG NURSES IN TAIWAN
}

\author{
YU-HUI HSIEH ${ }^{1,2}$, HSIU-HUNG WANG ${ }^{2}$, and SHU-CHING MA ${ }^{3}$ \\ ${ }^{1}$ Chia-Yi Christian Hospital, Ditmanson Medical Foundation, Chia-Yi, Taiwan \\ Center of Quality Management \\ ${ }^{2}$ Kaohsiung Medical University, Kaohsiung, Taiwan \\ College of Nursing \\ ${ }^{3}$ Chi-Mei Medical Center, Tainan, Taiwan \\ Department of Nursing
}

\begin{abstract}
Objectives: The aim of the study was to examine the relationship between workplace bullying, mental health and an intention to leave among nurses, and the mediating role of self-efficacy. Material and Methods: This cross-sectional study was conducted among 385 nurses in Taiwan. Data were collected by means of self-report questionnaires, including the Negative Acts Questionnaire-Revised, the General Self-Efficacy Scale, the Chinese Health Questionnaire, and the Employee's Turnover Intentions and Job Destination Choices Scale. Descriptive statistics, Pearson's correlation and hierarchical regression analyses were used. Results: Bullying was found to negatively correlate with self-efficacy and mental health, and positively with an intention to leave. Self-efficacy positively correlated with mental health, and negatively with an intention to leave. Hierarchical regression showed that bullying and self-efficacy were significant predictors of both mental health and an intention to leave. Self-efficacy partially mediated the relationship between bullying and mental health, as well as an intention to leave. Conclusions: Self-efficacy acted as a mediator of workplace bullying, mental health and an intention to leave among nurses. It could protect victims from the devastating effects of bullying behaviors. Int J Occup Med Environ Health. 2019;32(2):245-54
\end{abstract}

Key words:

workplace bullying, mental health, intention to leave, self-efficacy, nurses, Taiwan

\section{INTRODUCTION}

Workplace bullying (WPB) is a widespread issue recognized in many countries [1,2]. Employees in the health and social work sectors reported the highest levels of bullying, with nurses being vulnerable targets for workplace violence (WPV) [1,3]. A 2007 report of the International Council of
Nurses (ICN) indicated that $30.9 \%$ of nurses in Bulgaria were bullied, and so were $20.6 \%$ of nurses in South Africa and $10.5 \%$ in Australia [4]. Other studies have reported that $31-86 \%$ of nurses in different countries experience bullying in their workplace [5-7]. In Taiwan, 32.1-85\% of nurses reported that they experienced bullying [8-10].

Received: January 23, 2018. Accepted: July 11, 2018.

Corresponding author: Hsiu-Hung Wang, Kaohsiung Medical University, College of Nursing, 100 Shih-Chuan 1st Road, Kaohsiung City 80708, Taiwan (e-mail: hhwang@kmu.edu.tw). 
Bullying is defined as repeated negative treatment by one or more persons for a sustained period during which the person exposed to such treatment has difficulty defending $\mathrm{him} /$ herself against the perpetrators $[9,11]$.

Nurses worldwide have similar problems with bullying, which is known to have devastating effects on the victims' health, nursing profession, safety and quality of patient care, as well as on working environment in healthcare sectors, retention of nurses, and employers' costs [9,12-18]. Workplace bullying has been linked to negative health outcomes and mental health problems; bullied nurses are more likely to have headaches, hypertension, intestinal problems, fatigue, sleep disturbances, anxiety, irritability, depression, psychological distress and burnout, compared with non-bullied nurses [5,10,17-23]. In addition, many victims suffer from post-traumatic stress disorder (PTSD), which is reported to be the most adverse effect $[13,24]$. Bennett and Sawarzky [19] have found that WPB not only increases reports of psychosomatic complaints and psychological manifestations but also causes ineffective coping skills and may lead to impaired work relationships. Nurses who experience bullying are more likely to leave, or develop an intention to leave, their organization and the nursing profession entirely $[4,8,9,25,26]$. In 1 study, $29 \%$ of the victims reported voluntarily leaving their job in order toto discontinue their exposure to WPB [27].

Mikkelsen and Einarsen [28] have stated that general selfefficacy moderates the relationship between exposure to bullying behaviors and psychological health complaints, thereby protecting nurses who are exposed to bullying behaviors in the workplace from its adverse effects. Self-efficacy is defined as a person's self-confidence or belief in his or her capability to act and perform tasks in a particular situation; people can enhance their self-efficacy through personal experiences, the experiences of others, or a direct observation of others [29]. Newly graduated nurses, when exposed to bullying, tend to experience greater anxiety, lower self-esteem and lower self-confidence [30]. For experienced nurses, higher self-efficacy could diminish the impact of bullying on PTSD [24]. Studies have shown a strong association between personal self-efficacy and the performance level. The higher the self-efficacy, the more capable a person is when facing difficulties and overcoming obstacles, and, consequently, the more convinced he/she is about his/her ability to complete a difficult task [31,32]. Indeed, personal perceptions of selfefficacy affect the willingness to engage in certain behaviors and emotional responses to these behaviors (e.g., stress, anxiety and depression) [31]. Avey et al. [33] have found that psychological capital (PsyCap), which has the following 4 components: hope, resilience, optimism and self-efficacy, has significant positive effects on health and well-being in the workplace. They have also found that PsyCap partially mediates the effects of job stress on turnover intentions; employees with higher PsyCap tend to experience lower stress and are less likely to leave their jobs.

Thompson and George [34] have implemented an online educational module, revealing an association between module performance and self-efficacy scores; the student nurses indicated feeling more confident in avoiding becoming targets of bullying. Griffin [35] has proposed a cognitive rehearsal strategy in which nurses are taught to delay automatic thoughts and respond differently through empowerment strategies to address WPB; the participants perceived themselves to be more confident in their ability to recognize and address bullying in the workplace following their education. Indeed, there is a large body of literature confirming that higher self-efficacy can increase an individual's confidence to avoid becoming a target of bullying and, specifically, to protect nurses who are exposed to bullying behaviors in the workplace, with a view to diminishing the impact of bullying on their physical and mental health. However, existing research on WPB has paid little attention to the factors that could protect victims from the adverse effects of bullying. Therefore, this study aimed to: - examine the relationship between WPB, mental health and an intention to leave among nurses in Taiwan; 
- identify the protective factors against WPB to suggest interventions for nurses.

The following hypotheses were proposed:

- Hypothesis 1a: Exposure to WPB negatively correlates with mental health.

- Hypothesis 1b: Exposure to WPB positively correlates with an intention to leave.

- Hypothesis 2a: Self-efficacy mediates the relationship between WPB and mental health.

- Hypothesis 2b: Self-efficacy mediates the relationship between WPB and an intention to leave.

\section{MATERIAL AND METHODS}

\section{Design, setting and participants}

This cross-sectional study was conducted from October to December 2016. With convenience sampling, participants were recruited from a large teaching hospital in Taiwan with 1074 beds and a total of 720 nurses. The inclusion and exclusion criteria were as follows: being employed for at least 6 months in the hospital and holding permanent management or supervisory responsibilities, respectively. A total of 550 eligible nurses were invited to participate in this study. The participants' information was confidential and anonymous.

\section{Instruments}

The following instruments were employed using published scales that exhibited satisfactory validity and reliability in previous studies: the Negative Acts Questionnaire-Revised (NAQ-R), the General Self-Efficacy Scale (GSE), the Chinese Health Questionnaire (CHQ-12), and the Employee's Turnover Intentions and Job Destination Choices Scale.

\section{NAQ-R}

Bullying was measured using NAQ-R. Einarsen et al. [36] evaluated validity by examining the total NAQ-R, the General Health Questionnaire, and psychosomatic complaints for associations, which were moderately strong and statistically significant $(r=0.86, \mathrm{p}<0.001)$. NAQ-R was originally developed in English with Cronbach's $\alpha$ of 0.97 [37]. For the purpose of this study, it was translated into the participants' native language (Chinese). NAQ-R contains 22 items with 3 dimensions:

- personal bullying (12 items; e.g., being humiliated or ridiculed in connection with your work),

- work-related bullying (7 items; e.g., someone withholding information that affects your performance),

- physically intimidating forms of bullying ( 3 items; e.g., being shouted at or being a target of spontaneous anger).

The participants were asked to rate each item on a 5-point Likert scale: 1 - never, 2 - every now and then, 3 - monthly, 4 - weekly, and 5 - daily. Negative behavior intensity was measured by the sum of the total scores, within a range of 22-110. A higher score indicated a higher intensity of bullying behaviors. Cronbach's $\alpha$ of NAQ-R was 0.96 in this study.

The study also measured self-labeled exposure to bullying behavior in the past 6 months. The respondents were asked to indicate whether they considered themselves targets of bullying at work, according the given definition of bullying, and the item was rated using dichotomous "yes or no" response options.

\section{GSE}

This subscale of the overall self-efficacy scale was used to assess self-efficacy of the participants; Cronbach's $\alpha$ was 0.86 [38]. It was translated and revised by Huang and Cheng [39]; they evaluated its validity by examining its associations with self-concept, which were moderately strong and statistically significant $(\mathrm{r}=0.70, \mathrm{p}<0.001)$, with Cronbach's $\alpha$ of 0.88 . The 17-item GSE subscale (e.g., "I give up on things before completing them;" "I am a self-reliant person") was rated on a 6-point Likert scale from 1 - strong disagreement to 6 - strong agreement. The total scores were 17-102; the higher the score, the higher the self-efficacy expectations [33]. In this study, Cronbach's $\alpha$ of GSE was 0.91. 


\section{CHQ-12}

The questionnaire was used to measure mental health of the participants within the last 2 weeks [40]; its area under the ROC curve (AUC) values were $0.85 \pm 0.02$ with excellent discriminant validity and Cronbach's $\alpha$ of 0.84 [41]. The questionnaire contained items regarding the general physical status, depression, anxiety and sleep (e.g., losing much sleep due to worrying; losing confidence in oneself). Each item was rated on a 4-point Likert scale: 1 - not at all, 2 - same as usual, 3 - rather more than usual, and 4 - much more than usual; the responses "not at all" and "same as usual" were coded as 0 , whereas "rather more than usual" and "much more than usual" as 1 . The total scores were $0-12$; the higher the score, the higher the level or severity of mental health problems. In this study, Cronbach's $\alpha$ of CHQ-12 was 0.84 .

\section{Employee's Turnover Intentions and Job Destination Choices Scale}

An intention to leave was measured using the 5-item Employee's Turnover Intentions and Job Destination Choices Scale, with Cronbach's $\alpha$ of 0.71 [42]. Each item was rated on a 5-point Likert scale, ranging from 1 - strong disagreement to 5 - strong agreement. The total scores were 5-25, with a higher score indicating a greater intention to leave. In this study, Cronbach's $\alpha$ for this tool was 0.81 .

\section{Data analysis}

Data analysis was performed using SPSS 18.0 (SPSS Inc., Chicago, USA). Descriptive statistics were used to identify the demographic characteristics of the participants: age, marital status, children, education and religion. Pearson's correlation was used to examine the correlations between bullying, mental health, an intention to leave and self-efficacy. The present research used the Baron and Kenny approach [43] to examine self-efficacy as a potential mediator with respect to the association between bullying and mental health, as well as intention to leave factors. In conducting all the analyses, the significance level was set at 0.05 .

\section{Ethical considerations}

The Ethics Committee's approval was obtained from the Institutional Review Board (IRB) of the research institution (IRB No. 105040).

\section{RESULTS}

\section{Descriptive statistics and correlations}

A total of 550 eligible nurses were invited to participate in this study. A total of 442 participants returned their questionnaires, which yielded a response rate of $80.4 \%$. Of these, 385 questionnaires were complete and valid. The mean age in the sample $(\mathrm{N}=385)$ was $29.50( \pm 6.15)$, with a range of $21-50$. The majority of the sample (81\%) had completed a bachelor's degree. The average tenure in nursing was $7.28 \pm 6.12$ years (Table 1 ).

Table 1. Demographic characteristics of the participants $(\mathrm{N}=385)$ of the study on workplace bullying and self-efficacy among nurses in Taiwan

\begin{tabular}{lcc}
\hline \multicolumn{1}{c}{ Variable } & $\mathrm{n}(\%)$ & $\mathrm{M} \pm \mathrm{SD}$ \\
\hline Age [years] & & $29.50 \pm 6.15$ \\
Marital status & & \\
$\quad$ married or living with a partner & $112(29.1)$ & \\
$\quad$ single & $273(70.9)$ & \\
Children & & \\
$\quad$ yes & $96(24.9)$ & \\
no & $289(75.1)$ & \\
Education & & \\
$\quad \geq$ college & $311(81.0)$ & \\
$\quad<$ college & $73(19.0)$ & \\
Religious beliefs & \\
yes & $239(63.1)$ & \\
$\quad$ no & $140(36.9)$ & \\
Tenure in nursing work [years] & \\
Exposure to bullying behavior & \\
yes & $82(21.3)$ & \\
no & $298(78.4)$ & \\
\hline
\end{tabular}


Table 2. Means, standard deviations and correlations for the variables $(\mathrm{N}=385)$ in the study on workplace bullying and self-efficacy among nurses in Taiwan

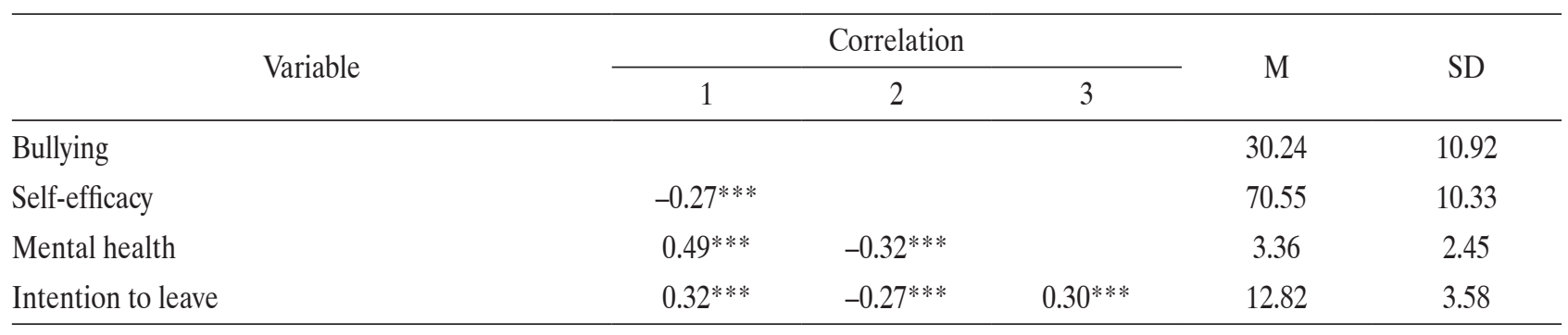

${ }^{*} \mathrm{p}<0.05 ;{ }^{* *} \mathrm{p}<0.01 ; * * \mathrm{p}<0.001$.

Correlation analysis (Table 2) showed that bullying was positively correlated with mental health $(r=0.49$, $\mathrm{p}<0.001)$ and an intention to leave $(\mathrm{r}=0.32, \mathrm{p}<0.001)$, and negatively with self-efficacy $(r=-0.27, \mathrm{p}<0.001)$. Self-efficacy was negatively correlated with mental health $(\mathrm{r}=-0.32, \mathrm{p}<0.001)$ and an intention to leave $(\mathrm{r}=-0.27$, $\mathrm{p}<0.001)$. Mental health was positively correlated with an intention to leave $(r=0.30, p<0.001)$.

\section{Mediating effect of self-efficacy on the relationship} between bullying, mental health and an intention to leave

To examine the hypothesis that self-efficacy is a protective factor in the relationship between WPB, mental health (Model 1) and an intention to leave (Model 2), the authors conducted a hierarchical regression analysis; the results are given in Table 3. Model 1.1 showed bullying as being positively associated with mental health $(\beta=0.49$, $\mathrm{p}<0.001$ ). In Model 1.2, self-efficacy was shown to account for a significant portion of variance in mental health $(27.6 \%, \mathrm{p}<0.001)$. Additionally, self-efficacy was negatively associated with mental health $(\beta=-0.21, \mathrm{p}<0.001)$. Model 2.1 showed bullying as positively associated with an intention to leave $(\beta=0.32, p<0.001)$. Model 2.2 demonstrated self-efficacy accounting for a significant portion of variance in an intention to leave $(13.5 \%, \mathrm{p}<0.001)$, while self-efficacy was negatively associated with an intention to leave $(\beta=-0.20, p<0.001)$.

In step 1 , bullying was a significant predictor of mental health $(\beta=0.49, \mathrm{p}<0.001)$ and an intention to leave $(\beta=0.32, p<0.001)$. In step 2 , bullying significantly predicted self-efficacy $(\beta=-0.27, p<0.001)$. In step 3 , the bullying/mental health path was lower (but significant)

Table 3. Results of hierarchical regression analyses in the study on workplace bullying and self-efficacy among nurses in Taiwan

\begin{tabular}{lcccc}
\hline & \multirow{2}{*}{ Variable } & \multicolumn{2}{c}{ Model 1 } & \multicolumn{2}{c}{ Model 2 } \\
& \multicolumn{2}{c}{ Mental health } & \multicolumn{2}{c}{ Intention to leave } \\
\cline { 2 - 5 } & model 1.1 & model 1.2 & model 2.1 & model 2.2 \\
\hline NAQ-R score & $0.49^{* * * *}$ & $0.44^{* * *}$ & $0.32^{* * *}$ & $0.27^{* * *}$ \\
Self-efficacy & & $-0.21^{* * *}$ & & $-0.20^{* * *}$ \\
$\mathrm{~F}$ & $119.71^{* * *}$ & $72.89^{* * *}$ & $44.07^{* * *}$ & $30.79^{* * *}$ \\
$\mathrm{R}^{2}$ & 0.24 & 0.28 & 0.11 & 0.14 \\
Adjusted R $^{2}$ & 0.24 & 0.28 & 0.10 & 0.14 \\
$\Delta \mathrm{R}^{2}$ & & 0.04 & & 0.03 \\
\hline
\end{tabular}

$* \mathrm{p}<0.05 ; * \mathrm{p}<0.01 ; * * \mathrm{p}<0.001$. 
compared with step $1(\beta=0.44, \mathrm{p}<0.001)$, suggesting partial mediation; the bullying/intention to leave path was lower (but significant) compared with step $1(\beta=0.27$, $\mathrm{p}<0.001$ ), also suggesting partial mediation.

\section{DISCUSSION}

In this study, $21.3 \%$ of the participants had experienced WPB in the past 6 months. This figure is close to the prevalence of bullying (32.1\% to $35.5 \%$ ) in previous works that used the self-labeling method to collect data [5,9], but it is significantly lower than that in studies which used the behavioral experience method ( $85 \%$ to $86 \%$ ) [6,8]. Nielsen et al. [44] mentioned that the approach to explore the prevalence of WPB includes the behavioral experience and self-labeling methods. As such, significant differences in the reported prevalence of bullying can be attributed to the investigative method used. The self-labeling method tends to yield lower prevalence compared with the behavioral experience method. Specifically, different measurement tools and operational concepts of bullying will result in different prevalence rates.

In the present study, the results support hypotheses $1 \mathrm{a}$ and $1 \mathrm{~b}$. The findings are consistent with previous research linking bullying to numerous negative health outcomes; bullied nurses are more likely to have physical and mental health problems compared with non-bullied nurses [9,1720]. Those participants who reported greater exposure to WPB were more likely to have a greater intention to leave. This finding is similar to that in previous studies $[8,9,16,26]$.

The major purpose of this study was to examine the role of self-efficacy in protecting nurses exposed to WPB. The results also supported hypotheses $2 \mathrm{a}$ and $2 \mathrm{~b}$. We demonstrated that self-efficacy could protect nurses exposed to WPB, helping them avoid the adverse effects on their health outcomes, and decrease their intention to leave. This result corresponded with that presented by Laschinger and Nosko [24] who found that the impact of bully- ing on mental health - PTSD - was stronger in nurses with lower levels of self-efficacy. Additionally, the present findings confirm the reports in Mikkelsen and Einarsen [28] on the moderating role of self-efficacy in the relationship between bullying and the subsequent health complaints.

We recommend the development and evaluation of relevant interventions that can enhance self-efficacy in individuals, which will help them cope with stressful situations and mitigate the negative effects of WPB. Simultaneously, medical institutions should establish an easy-to-use notification system through which victims could feel free to report any WPB experience promptly and in a safe manner. Further, institutions should provide the necessary education and counseling resources, as well as support group for victims, promote personal self-efficacy and reduce the harmful effects of WPB on victims.

Moreover, medical institutions should establish a policy and workplace environment for zero tolerance for bullying, as is the position of the Center for American Nurses. In this way, they can provide a safe and friendly environment for nurses. Nurses who face unequal treatment at work, especially where the perpetrators hold a higher position, have reported the feeling of having no chance to change anything, thereby opting for a passive stance to avoid conflict or even choosing to stay silent on the issue $[9,10]$. Supervisors or managers should have the ability of self-reflection to examine whether the leadership is appropriate, and pay attention to inappropriate behavior or bullying behavior in the organization or institution, which should be dealt with as early as possible to avoid bullying from spreading or encouraging the atmosphere of bullying. The institution should provide nurses with education and training to increase their self-efficacy and confidence [34], especially as regards new nurses [29], including identifying bullying behaviors, preventing bullying and strengthening individual self-efficacy. As stated, individuals can enhance their self-efficacy through personal experiences, the experiences of others, or direct ob- 
servation of others [30]. Particularly, nurses can practice communicating with others, dealing with negative emotions, coping with stressful situations, practicing a cognitive rehearsal strategy to delay automatic thoughts, and responding differently through empowerment strategies. Through such educational opportunities, nurses could distinguish and address disruptive behaviors by others, increase the perception of WPB, and increase their personal self-efficacy. Finally, nurses who face bullying in the workplace should be encouraged to notify their organization to stop bullying behaviors and take a proactive role in changing the culture of silence on organizational bullying.

\section{Limitations}

The study has several limitations. First, the authors adopted a cross-sectional design, which limits the extent to which causal relationships can be inferred. Second, the sample was collected from only 1 hospital in this study, and as such, the results must be generalized with caution. Third, the data were self-reported, thereby carrying a risk of recall bias; the attitude and response of the participants would be affected by social desirability. Finally, although the response rate was $80.4 \%$ in this study, the authors could not obtain the demographic characteristics and reasons for incomplete answers for the remaining 19.6\% of the respondents, or verify whether they suffered from WPB in the last 6 months.

For future research, a longitudinal study that collects data from different periods and hospitals would be better to test and establish the direction of the relationships between the research variables. In addition to the selfreport, other information sources, such as organizational reports and personal in-depth interviews, could be adopted. Using online questionnaires might increase the privacy and reduce the interference from others, as well as increase the willingness of the participants to respond candidly.

\section{CONCLUSIONS}

This study examined the relationship among WPB, mental health and an intention to leave among nurses, and extended the previous research by emphasizing the protective factors of self-efficacy that protect nurses exposed to WPB from its harmful consequences. The findings showed that bullying and self-efficacy were significant predictors of mental health, as well as an intention to leave. Selfefficacy partially mediated the relationship between bullying and mental health, and an intention to leave among nurses. Future research is necessary to explore the nature, causes and protective factors of WPB, to help reduce its harmful consequences. Most importantly, relevant interventions need to be developed to enhance individuals' self-efficacy to help them cope with stressful situations and buffer the negative effects of WPB.

\section{ACKNOWLEDGMENTS}

We appreciate the participating hospital and all of the nurses who participated in this study.

\section{REFERENCES}

1. European Foundation for the Improvement of Living and Working Conditions. Physical and psychological violence at the workplace [Internet]. Dublin: The Foundation; 2010 [cited 2017 Sep 3]. Available from: http://www.eurofound.europa.eu/pubdocs/2010/54/en/1/EF1054EN.pdf.

2. Namie G. 2014 WBI U.S. workplace bullying survey [Internet]. Clarkston: Workplace Bullying Survey; 2014 [cited 2017 Sep 3]. Available from: http://www.workplacebullying.org/ multi/pdf/WBI-2014-US-Survey.pdf.

3. Roberts S, Demacro R, Griffin, M. The effect of oppressed group behaviours on the culture of nursing workplace: A review of the evidence and interventions for change. J Nurs Manag. 2009;17(3):288-93, https://doi.org/10.1111/j.1365-2834.2008. 00959.x.

4. International Council of Nurses. Guidelines on coping with violence in the workplace [Internet]. Geneva: The Council; 
2007 [cited 2017 Sep 3]. Available from: http://www.icn. ch/images/stories/documents/pillars/sew/sew_framework guidelines_for_addressing_workplace_violence.pdf.

5. Rodwell J, Demir D, Steane P. Psychological and organizational impact of bullying over and above negative affectivity: A survey of two nursing contexts. Int J Nurs Pract. 2013;19(3):241-8, https://doi.org/10.1111/ijn.12065.

6. Yildirim A, Yildirim D. Mobbing in the workplace by peers and managers: Mobbing experienced by nurses working in health care facilities in Turkey and its effect on nurses. J Clin Nurs. 2007;16(8):1444-53, https://doi.org/10.1111/j.1365-2702. 2006.01814.x.

7. Simons S. Workplace bullying experienced by Massachusetts registered nurses and the relationship to intention to leave the organization. Adv Nurs Sci. 2008;31(2):E48-59, https:// doi.org/10.1097/01.ANS.0000319571.37373.d7.

8. Tsai ST, Han CH, Chen LF, Chou FH. [Nursing workplace bullying and turnover intention: An exploration of associated factors at a medical center in Southern Taiwan]. J Nurs. 2014;61(3):58-68. Chinese.

9. Ma SC. [Current situation and related factors of workplace bullying among hospital nurses]. [unpublished doctoral dissertation]. Kaohsiung: Kaohsiung Medical University; 2014. Chinese.

10. Pai HC, Lee S. Risk factors for workplace violence in clinical registered nurses in Taiwan. J Clin Nurs. 2011;20(9-10):140512, https://doi.org/10.1111/j.1365-2702.2010.03650.x.

11. Einarsen S, Hoel H, Zapf D, Cooper CL. Bullying and harassment in the workplace. 2nd ed. Boca Raton: CRC Press, Taylor and Francis Group; 2011.

12. American Nurses Association [Internet]. Silver Spring: ANA Enterprise; 2015 [cited 2017 Sep 3]. Incivility, bullying, and workplace violence. Available from: http://www.nursingworld. org/MainMenuCategories/Policy-Advocacy/Positions-andResolutions/ANAPositionStatements/Position-Statements-Alphabetically/Incivility-Bullying-and-Workplace-Violence.html.

13. Bigony L, Lipke TG, Lundberg A, McGrew CA, Pagac GL, Rogers A. Lateral violence in the perioperative setting.
AORN J. 2009;89(4):688-700, https://doi.org/10.1016/j.aorn. 2009.01.029.

14. Laschinger HKS. Impact of workplace mistreatment on patient safety risk and nurse-assessed patient outcome. J Nurs Adm. 2014;44(5):284-90, https://doi.org/10.1097/NNA. 0000000000000068.

15. Lewis PS, Malecha A. The impact of workplace incivility on the work environment, manager skill, and productivity. J Nurs Adm. 2011;41(1):41-7, https://doi.org/10.1097/ NNA.0b013e3182002a4c.

16. Longo J. Bullying in the workplace: Reversing a culture. Silver Spring: American Nurses Association/Nursebooks.org; 2012.

17. Namie G. The WBI Website 2012 Instant Poll D - Impact of Workplace Bullying on Individuals' Health [Internet]. Clarkstone: Workplace Bullying Institute; 2012 [cited 2017 Sep 3]. Available from: http://www.workplacebullying.org/ multi/pdf/WBI-2012-IP-D.pdf.

18. Yildirim D. Bullying among nurses and its effects. Int Nurs Rev. 2009;56(4):504-11, https://doi.org/10.1111/j.1466-76 57.2009.00745.x.

19. Bennett K, Sawarzky J. Building emotional intelligence: A strategy for emerging nurse leaders to reduce workplace bullying. Nurs Adm Q. 2013;37(2):144-51, https://doi.org/ 10.1097/NAQ.0b013e318286de5f.

20. Hauge LJ, Skogstad A, Einarsen S. The relative impact of workplace bullying as a social stressor at work. Scand J Psychol. 2010;51(5):426-33, https://doi.org/10.1111/j.14679450.2010.00813.x.

21. Laschinger HKS, Grau AL. The influence of personal dispositional factors and organizational resources on workplace violence, burnout, and health outcomes in new graduate nurses: A cross-sectional study. Int J Nurs Stud. 2014;49(3):28291, https://doi.org/10.1016/j.ijnurstu.2011.09.004.

22. Rodwell J, Demir D. Psychological consequence of bullying for hospital and aged care nurses. Int Nurs Rev. 2012;59(4):539-46, https://doi.org/10.1111/j.1466-7657.2012. 01018.x. 
23. Vessey JA, Demarco RF, Gaffney DA, Budin WC. Bullying of staff registered nursing in the workplace: A preliminary study for developing personal and organizational strategies for the transformation of hostile to healthy work environments. J Prof Nurs. 2009;25(5):299-306, https://doi. org/10.1016/j.profnurs.2009.01.022.

24. Laschinger HKS, Nosko A. Exposure to workplace bullying and post-traumatic stress disorder symptomology: The role of protective psychological resources. J Nurs Manag. 2015; 23(3):252-62, https://doi.org/10.1111/jonm.12122.

25. Hauge LJ, Skogstad A, Einarsen S. Relationships between stressful work environments and bullying: Results of a large representative study. Work Stress. 2007;21(3):220-42.

26. Johnson SL, Rea RE. Workplace bullying: Concerns for nurse leaders. J Nurs Adm. 2009;39(2):84-90, https://doi. org/10.1097/NNA.0b013e318195a5fc.

27. Clausen T, Hogh A, Carneiro IG, Borg V. Does psychological well-being mediate the association between experiences of acts of offensive behaviour and turnover among care workers? A longitudinal analysis. J Adv Nurs. 2013;69(6):130113, https://doi.org/10.1111/j.1365-2648.2012.06121.x.

28. Mikkelsen EG, Einarsen S. Relationships between exposure to bullying at work and psychological and psychosomatic health complaints: The role of state negative affectivity and generalized self-efficacy. Scand J Psychol. 2002;43(5):397405, https://doi.org/10.1111/1467-9450.00307.

29. Bandura A. Self-efficacy: The exercise of control. New York: Freeman; 1986.

30. McKenna BG, Smith NA, Poole SJ, Coverdale JH. Horizontal violence: Experiences of registered nurses in their first year of practice. J Adv Nurs. 2003;42(1):90-6, https://doi. org/10.1046/j.1365-2648.2003.02583.x.

31. Bandura A. Self-efficacy: Toward a unifying theory of behaviour change. Psychol Rev. 1977;84(2):191-215, https://doi. org/10.1037//0033-295X.84.2.191.

32. Bandura A. Self-efficacy mechanism in human agency. Am Psychol. 1982;37(2):122-47, https://doi.org/10.1037//0003066X.37.2.122.
33. Avey JB, Luthans F, Jensen SM. Psychological capital: A positive resource for combating employee stress and turnover. Hum Resour Manag. 2009;48(5):677-93, https://doi. org/10.1002/hrm.20294.

34. Thompson R, George LE. Preparing new nurses to address bullying: The effect of an online educational module on learner self-efficacy. Medsurg Nurs. 2016;25(6):133-57.

35. Griffin M. Teaching cognitive rehearsal as a shield for lateral violence: An intervention for newly licensed nurses. J Contin Educ Nurs. 2004;35(6):257-63.

36. Einarsen S, Hoel H, Notelaers G. Measuring exposure to bullying and harassment at work: Validity, factor structure, and psychometric properties of the Negative Acts Questionnaire-Revised. Work Stress. 2009;23(1):24-44.

37. Ma SC, Tsair WC, Wang HH, Li YC, Yui MS. Applying computerized adaptive testing to the Negative Acts Questionnaire-Revised: Rasch analysis of workplace bullying. J Med Internet Res. 2014;16(2):1-14, https://doi.org/10.2196/ jmir.2819.

38. Sherer M, Maddux JE, Mercandante B, Prentice-Dunn S, Jacobs B, Rogers RW. The self-efficacy scale: Construction and validation. Psychol Rep. 1982;51(2):663-71, https://doi. org/10.2466/pr0.1982.51.2.663.

39. Huang YH, Cheng YY. [The revision of general self-efficacy scale]. Psychol Test. 1996;43:279-86. Chinese.

40. Cheng TA, Williams P. The design and development of a screening questionnaire (CHQ) for use in community studies of mental disorders in Taiwan. Psychol Med. 1986;16(2):41522, https://doi.org/10.1017/S0033291700009247.

41. Cheng TA, Wu JT, Chong MY, Williams P. Internal consistency and factor structure of the Chinese Health Questionnaire. Acta Psychiatr Scand. 1990;82(4):304-8, https://doi. org/10.1111/j.1600-0447.1990.tb01389.x.

42. Kirschenbaum A, Weisberg J. Employee turnover intentions and job destination choices. J Organ Behav. 2002;23(1):10925, https://doi.org/10.1002/job.124.

43. Baron RM, Kenny DA. The moderator-mediator variable distinction in social psychological research: Conceptual, 
strategic, and statistical considerations. J Pers Social Psychol. 1986;51:1173-82, https://doi.org/10.1037/0022-3514.51. 6.1173.

44. Nielsen MB, Matthiesen SB, Einarsen S. The impact of methodological moderators on prevalence rates of workplace bullying: A meta-analysis. J Occup Organ Psychol. 2010;83(4):955-79, https://doi.org/10.1348/096317909 X481256.

This work is available in Open Access model and licensed under a Creative Commons Attribution-NonCommercial 3.0 Poland License - http://creativecommons.org/ licenses/by-nc/3.0/pl/deed.en. 